\title{
Dipolar origin of the gas-liquid coexistence of the hard-core 1:1 electrolyte model
}

\author{
J. M. Romero-Enrique, ${ }^{1, *}$ L. F. Rull, ${ }^{1}$ and A. Z. Panagiotopoulos ${ }^{2}$ \\ ${ }^{1}$ Departamento de Física Atómica, Molecular y Nuclear, Área de Física Teórica, Universidad de Sevilla, \\ Apartado Correos 1065, 41080 Sevilla, Spain \\ ${ }^{2}$ Department of Chemical Engineering, Princeton University, Princeton, New Jersey 08544
}

(Received 26 June 2002; published 9 October 2002)

\begin{abstract}
We present a systematic study of the effect of the ion pairing on the gas-liquid phase transition of hard-core 1:1 electrolyte models. We study a class of dipolar dimer models that depend on a parameter $R_{c}$, the maximum separation between the ions that compose the dimer. This parameter can vary from $\sigma_{ \pm}$that corresponds to the tightly tethered dipolar dimer model to $R_{c} \rightarrow \infty$ that corresponds to the Stillinger-Lovett description of the free ion system. The coexistence curve and critical point parameters are obtained as a function of $R_{c}$ by grandcanonical Monte Carlo techniques. Our results show that this dependence is smooth but nonmonotonic and converges asymptotically towards the free ion case for relatively small values of $R_{c}$. This fact allows us to describe the gas-liquid transition in the free ion model as a transition between two dimerized fluid phases. The role of the unpaired ions can be considered as a perturbation of this picture.
\end{abstract}

DOI: 10.1103/PhysRevE.66.041204

PACS number(s): 64.70.Fx, 05.10.Ln, 05.70.Jk

\section{INTRODUCTION}

In recent years there has been an increasing interest in phase transitions between fluid phases of different electrolyte concentrations in ionic solutions. Two different regimes have been identified experimentally [1]. The "solvophobic" regime occurs for large solvent dielectric constants that effectively turn off Coulombic interactions. Consequently, solvophobic phase transitions are primarily driven by unfavorable interactions between solute and solvent. This behavior is well described by the usual theory of nonelectrolyte solutions with short-range interactions, and clearly leads to Isinglike critical behavior. By contrast, in the "Coulombic" regime the solvent has a low dielectric constant and electrostatic interactions between the solute ions drive the phase separation. In this case, the phase diagrams are quite asymmetric, and apparently mean-field critical behavior has been claimed [2-4], although Ising-mean-field crossover has also been seen within a narrower range of temperatures around the critical than in nonionic fluids [5-8]. Some of these experimental studies suggest that there exists a new characteristic length in these systems that competes with the correlation length for density fluctuations $[7,8]$.

Electrolyte systems in the Coulomb regime are often modeled as charged hard spheres embedded in a uniform dielectric continuum (primitive models). Most studied is the "restricted primitive model" (RPM), in which the ions are of equal size. A vapor-liquid phase transition at very low temperatures and densities was predicted theoretically 25 years ago [9] and by early computer simulation studies [10]. Improvement of computer simulation techniques has allowed an increasingly precise determination of the coexistence parameters [11-16]. There have also been recent studies of primitive models with asymmetry in size [17-19] and charge

\footnotetext{
*Corresponding author. Electronic address: enrome@us.es
}

$[19,20]$. Very recent results suggest that the critical behavior of the RPM belongs to the Ising universality class [16].

From a theoretical point of view, different approaches have been used in order to explain the vapor-liquid transition of the primitive models. Integral equations such as the mean spherical approximation [21,22], as well as the DebyeHückel theory [21] and Poisson-Boltzmann approaches [23] have succesfully been applied to it. For the RPM, the most succesful theories are the pairing theories that consider the ionic fluid as a mixture of bound pairs and free ions in chemical equilibrium $[21,24]$, in the spirit of the Bjerrum's ideas [25]. However, in all these theories the transition is driven by the free ions, even when in same cases, as in the Debye-Hückel-Bjerrum approach, the associated pairs are the dominant species. Analytical [26] and computer simulation [27] results, on the other hand, show that the structure of the vapor phase is dominated by neutral clusters, mostly dimers and tetramers. Computer simulations have also demonstrated that the phase envelope of the RPM resembles that of the charged hard dumbbell model $[28,17]$. This result has been confirmed by theoretical studies $[29,30]$. The question that the present work examines in detail is the influence of ionic association on the vapor-liquid transition of primitive model electrolytes. In contrast to an earlier study [17], which only considered tightly bound dimers, here we examine a range of models with varying values of $R_{c}$, the maximum separation between ions in a dimer. $R_{c} \rightarrow \infty$ corresponds to the Stillinger-Lovett description [31] of the free ion system.

The structure of this paper is as follows. In Sec. II, we examine the microscopic structure of the coexisting phases of the ionic fluid and compare the correlation functions to those of a tightly tethered dimer model. In Sec. III, an exact chemical representation of the ionic fluid as a mixture of associated pairs and free ions is introduced. The role of the associated pairs on the phase coexistence is studied in detail in Sec. IV and the paper closes with discussion and conclusions. 


\section{MICROSCOPIC STRUCTURE OF THE IONIC AND TETHERED DIMER SYSTEMS AT COEXISTENCE}

In this section we analyze the role of pairing on gas-liquid coexistence of primitive model ionic fluids. We have studied by computer simulation an 1:1 size-asymmetric primitive model, in which the ions are modeled as hard spheres of diameters $\sigma_{+}$and $\sigma_{-}$, carrying charges $+q$ and $-q$, respectively, embedded on a dielectric continuum of dielectric constant $D(D=1$ for the vacuum). The interaction potential between two ions separated by a distance $r_{i j}$ is given by

$$
u_{i j}\left(r_{i j}\right)=u_{h s}\left(r_{i j}\right)+\frac{q_{i} q_{j}}{D r_{i j}},
$$

where $u_{h s}\left(r_{i j}\right)$ is the hard-core potential that takes the value $+\infty$ if $r_{i j}<\frac{1}{2}\left(\sigma_{i}+\sigma_{j}\right)$ and 0 otherwise.

The size asymmetry is characterized by the parameter $\lambda$, defined as

$$
\lambda=\frac{\sigma_{-}}{\sigma_{+}} .
$$

Monte Carlo simulations in the neutral grand-canonical ensemble were performed, characterized by a temperature $T$ and the configurational chemical potential for a pair of unlike ions $\mu=2 \mu_{\text {real }}-3 k_{B} T \ln \left(\Lambda_{+} \Lambda_{-}\right)$, with $\mu_{\text {real }} \equiv\left(\mu_{+}\right.$ $\left.+\mu_{-}\right) / 2$ and $\Lambda_{ \pm}$being the thermal de Broglie wavelengths of the ionic species,

$$
\Lambda_{ \pm}=\sqrt{\frac{h^{2}}{2 \pi m_{ \pm} k_{B} T}}
$$

As usual, cubic boxes of length $L$ under periodic boundary conditions are used. The long-ranged character of the Coulombic interactions is handled by the use the Ewald summation technique with conducting boundary conditions, with 518 Fourier-space wave vectors and real-space damping parameter $\kappa=5$. The relative error due to the infinite sums truncation in the electrostatic energy is less than $10^{-5}$ for random configurations in small systems [32], and this choice has been also validated by direct simulations of the RPM [15]. In order to speed up the simulations, the basic steps of the simulations (insertion and deletions of pairs of unlike ions) are biased following Ref. [11]. Moreover, a finediscretization approximation is used: the positions available to each ion are the sites of a simple cubic lattice of spacing $a$. This methodology has succesfully been applied to the RPM [14], to 1:1 size-asymmetric primitive models [17], and to $z: 1$ size-asymmetric primitive models [14], and allows a speedup relative to the continuum calculations of a factor of 100 for small systems. The results are almost indistinguishable from the continuum ones for a discretization parameter $\zeta \equiv \sigma_{ \pm} / a=10[14,17,19]$, where $\sigma_{ \pm}=\frac{1}{2}\left(\sigma_{+}+\sigma_{-}\right)$is the unlike-ion collision diameter. This value of the discretization parameter $(\zeta=10)$ was used in the present study. Histogram reweighting techniques [33] and mixed-field finite-size scaling methods [34] were used to obtain the vapor-liquid envelopes and the effective critical points, respectively. For the sake of comparison, we have also studied tightly tethered

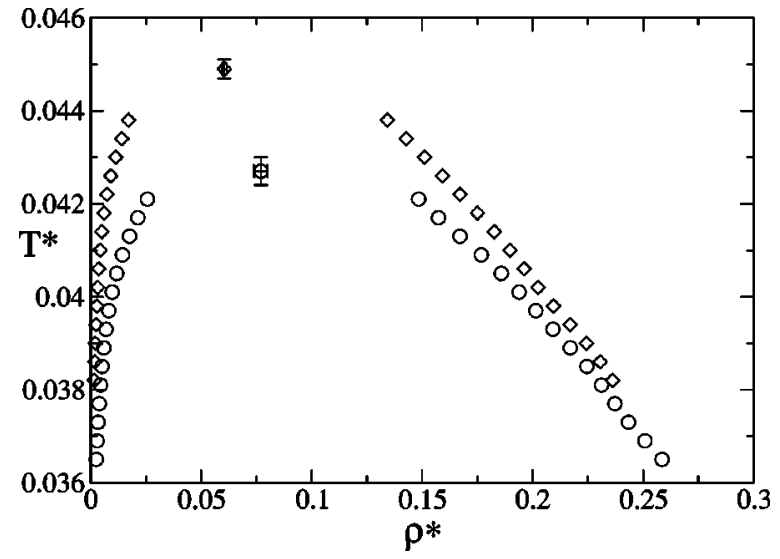

FIG. 1. Gas-liquid phase diagram for the ionic system (diamonds) and the tethered dimer fluid (circles) with a tether length equal to $1.02 \sigma_{ \pm}$. The size asymmetry parameter is set $\lambda=3$. The critical parameters have been obtained from the extrapolation to $L^{*} \rightarrow \infty$ of the results for $L^{*}=12,15$, and 18 while the subcritical coexistence curves were obtained using $L^{*}=15$.

dipolar dimer systems [17,28], consisting of $N$ pairs of a positive and a negative ion restricted to remain at separations $\sigma_{d}$ satisfying $\sigma_{ \pm} \leqslant \sigma_{d} \leqslant 1.02 \sigma_{ \pm}$. Simulation details and some preliminary results for a range of values of $\lambda$ are presented in Ref. [17].

The unlike-ion collision diameter $\sigma_{ \pm}$provides the basic length scale appropriate for defining both the reduced temperature and reduced density via

$$
T^{*}=k_{B} T D \sigma_{ \pm} / q^{2} \quad \text { and } \quad \rho^{*}=2 N \sigma_{ \pm}^{3}, / L^{3}
$$

where $N_{+}=N_{-} \equiv N$ is the particle number of each ionic species $[11,17,22]$. The reduced simulation box length is defined similarly via $L^{*}=L / \sigma_{ \pm}$, and the reduced energies and chemical potential as $U^{*}=U D \sigma_{ \pm} / q^{2}$ and $\mu^{*}$ $=\mu D \sigma_{ \pm} / q^{2}$.

The value of the asymmetry parameter considered in this paper is $\lambda=3$. For that case, the gas-liquid coexistence of the ionic fluid shows a shift in both temperature and density with respect to the tethered dimer fluid (see Fig. 1), which is a general feature when comparing ionic and tethered dimer systems [17]. On the other hand, the asymmetry is not high so as to favor large chainlike neutral clusters, as occurs for bigger values of $\lambda[17,18]$. These features qualify this case to be a typical example for moderately asymmetric 1:1 electrolytes, including the RPM.

Figure 1 makes the similarity between the phase diagram of the ionic and the tethered dimer fluids clear. It also suggests in an indirect way that the pairing plays a decisive role on the gas-liquid coexistence in the ionic fluid. In order to clarify such a role, we have studied the ion-ion radial distribution functions $g_{i j}(r)$ corresponding to the ionic systems, and the corresponding ones for the tethered dimer systems. For this purpose, we have considered the gas and liquid states at coexistence for a temperature $T \approx 0.95 T_{c}$, with $T_{c}$ being the corresponding critical temperature. The ion-ion radial distribution functions of the ionic systems, for $T^{*}$ $=0.0425$ and the coexisting densities $\rho^{*}=0.0076(15)$ and 


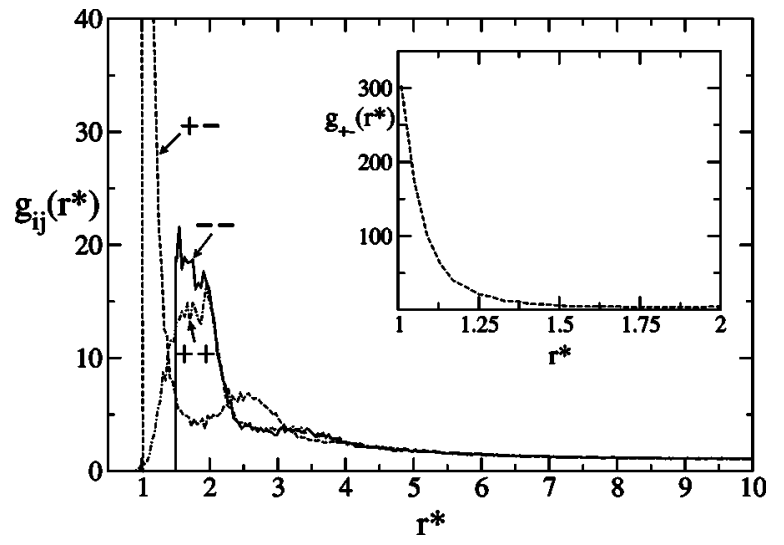

FIG. 2. Ion-ion radial distribution functions corresponding to the ionic system at $T^{*}=0.0425$ and $\rho^{*}=0.0076(15)$ (the gas phase). The inset shows the behavior of $g_{+-}\left(r^{*}\right)$ at distances close to the contact value.

$\rho^{*}=0.162(3)$ are plotted in Figs. 2 and 3, respectively. In both cases, the unlike-ion radial distribution function becomes very large close to contact, indicating the association in bound pairs of unlike ions. Moreover, the like-ion radial distribution functions show maxima around $r^{*} \equiv r \sigma_{ \pm}=1.5$ (in the case of $g_{--}$, this peak coincides with the contact value), and there is a secondary maximum in $g_{+-}$for $r^{*}$ $\approx 2.5$. These observations allow us to conclude that there is high correlation between pairs of associated unlike ions. We recall that the range of densities in which the gas-liquid coexistence occurs prevents packing effects, so the structure is completely given by the Coulombic interactions.

The ion-ion radial distribution functions for the tethered dimer fluid are plotted in Fig. 4 for the gas branch $\left[T^{*}\right.$ $\left.=0.0405, \rho^{*}=0.0119(15)\right]$ and in Fig. 5 for the liquid branch $\left[T^{*}=0.0405, \rho^{*}=0.206(2)\right]$. The comparison between the ionic fluid and tethered dimer fluid microscopic structures confirms the qualitative similarity between both systems. A further test of this similarity is found in the comparison of the neutral cluster populations in the gas branch. We use Gillan's definition of a cluster [26]. Two ions $i$ and $j$ are directly bound when the distance between them is less than $R_{i j}^{c}$. This condition defines mathematically an equiva-

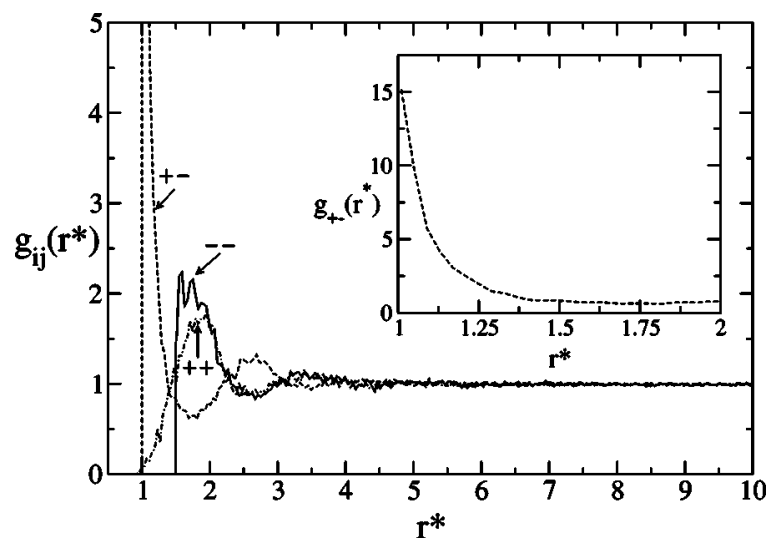

FIG. 3. Same as in Fig. 2 in the liquid branch: $T^{*}=0.0425$ and $\rho^{*}=0.162(3)$.

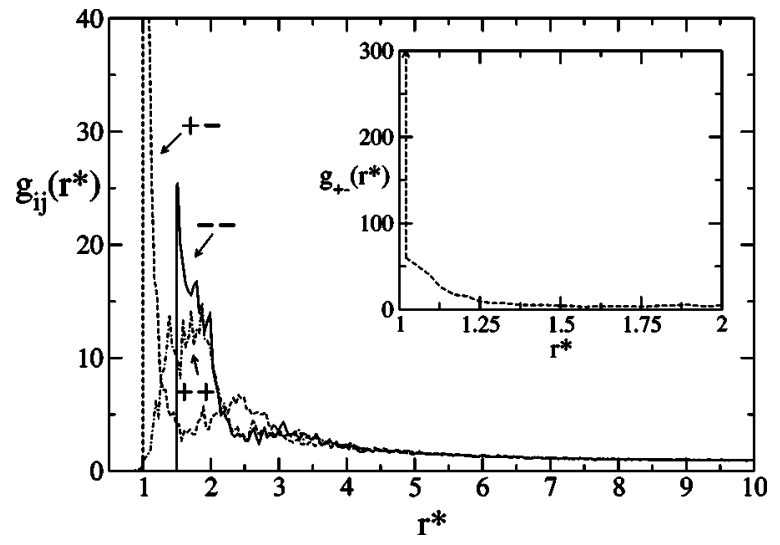

FIG. 4. Ion-ion radial distribution functions corresponding to the tethered dimer system at $T^{*}=0.0405$ and $\rho^{*}=0.0119$ (15) (the gas phase). The inset shows the behavior of $g_{+-}\left(r^{*}\right)$ at distances close to the contact value.

lence relationship, and the equivalences classes in which the ions group are the clusters. As the interaction between like ions is repulsive, the dependence of the cluster definition on $R_{++}^{c}$ or $R_{--}^{c}$ should be very weak (if they are taken smaller than the mean distance between two like ions). On the other hand, the cluster definition is not very sensitive to the value of $R_{+-}^{c}$ if that value lies between the first minimum of $g_{+-}$ and the mean distance between aggregates. In this work we have used $R_{++}^{c}=R_{--}^{c}=R_{+-}^{c}=1.5 \sigma_{ \pm}$. As expected, the microscopic structure of the gas phase is dominated by the $N$-ion neutral clusters $[17,27]$. Their fractions $f_{N}$ for the ionic and tethered ion systems at the gas phase in coexistence for $T=0.95 T_{c}$ are quite similar (see Fig. 6), although the tethered dimer systems have slightly higher fractions of neutral clusters than do ionic systems, specially for large $N$, in agreement with the results previously reported [17].

Despite the similarities found between the ionic fluid and the tethered dimer fluid, there are some differences that can rationalize the quantitative differences between their phase diagrams. The unlike-ion radial distribution functions of the tethered dimer fluid differ qualitatively from the ionic counterparts close to the contact value, since in the former there is a jump at the maximum allowed separation of two ions of a

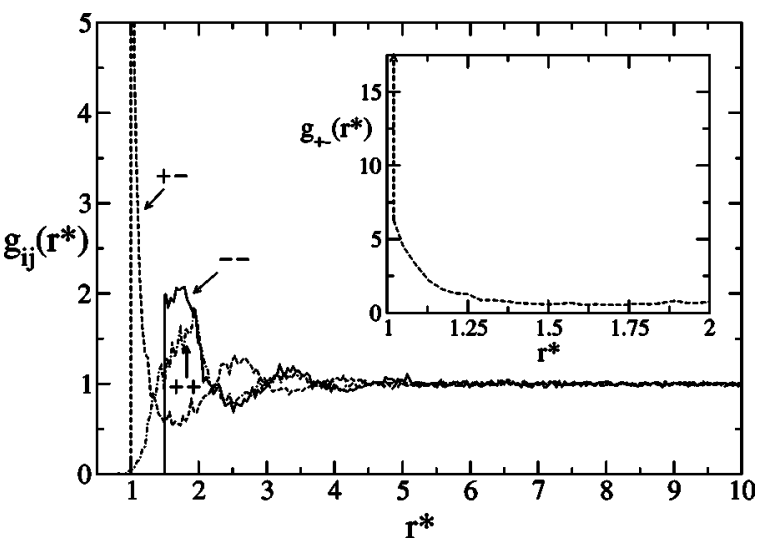

FIG. 5. Same as in Fig. 4 in the liquid branch: $T^{*}=0.0405$ and $\rho^{*}=0.206(2)$. 


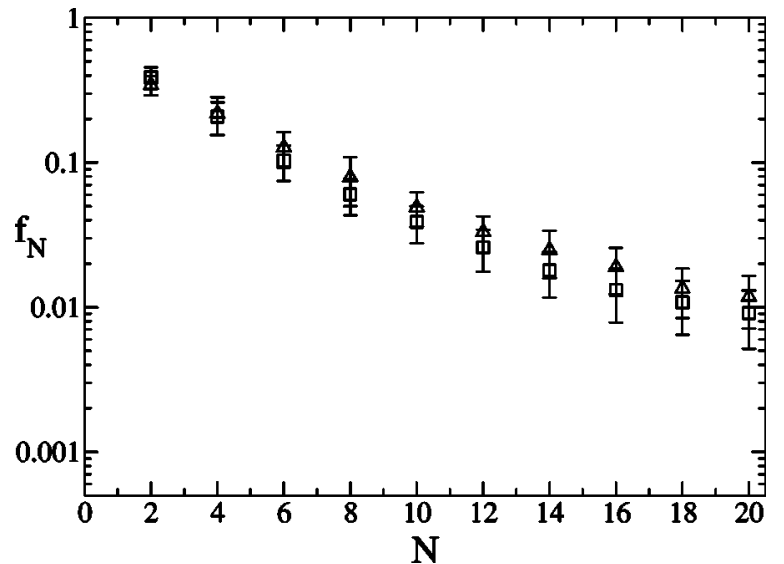

FIG. 6. Fraction $f_{N}$ of $N$-ion neutral clusters. The squares correspond to the ionic fluid at $T^{*}=0.0425$ and $\rho^{*}=0.0076(15)$, and the triangles to the tethered dimer fluid at $T^{*}=0.0405$ and $\rho^{*}$ $=0.0119(15)$.

dimer, while the ionic $g_{+-}$takes smoothly higher values on that range of values of $r^{*}$. Consequently, the condition on the confinement of the ions that compose a tethered dimer must be relaxed in order to refine the pairing concept in the ionic fluids. In the following section we will introduce an appropriate framework for such a goal.

\section{CHEMICAL PICTURE OF IONIC FLUIDS}

We consider an ionic fluid $\left(q_{+}=-q_{-} \equiv q\right)$ contained in a volume $V$ and in equilibrium with a reservoir at a temperature $T$ and chemical potentials $\mu_{+}$and $\mu_{-}$. For simplicity, the neutral grand-canonical ensemble, in which only neutral configurations are allowed, will be considered. This ensemble has been shown to be equivalent to the usual grandcanonical ensemble in the thermodynamic limit [35]. The neutral grand-canonical partition function can be written as

$$
\Xi=\sum_{N_{i}=0}^{\infty}\left(\frac{e^{\beta \mu}}{\Lambda_{+}^{3}}\right)^{N_{i}}\left(\frac{e^{\beta \mu}}{\Lambda_{-}^{3}}\right)^{N_{i}} Z_{U}\left(N_{i}, N_{i}\right)
$$

where $N_{i}$ is the number of ions of each species, $\beta$ $=1 /\left(k_{B} T\right), \mu \equiv\left(\mu_{+}+\mu_{-}\right) / 2, \Lambda_{+}$and $\Lambda_{-}$are the thermal de Broglie wavelengths corresponding to each species, and $Z_{U}\left(N_{i}, N_{i}\right)$ is the canonical configurational partition function corresponding to a fixed number of ions at the temperature $T$ and enclosed in the volume $V$,

$$
Z_{U}\left(N_{i}, N_{i}\right)=\frac{1}{\left(N_{i} !\right)^{2}} \int_{V^{2 N_{i}}} d \mathbf{r}_{1}^{+} d \mathbf{r}_{1}^{-} \ldots d \mathbf{r}_{N_{i}}^{+} d \mathbf{r}_{N_{i}}^{-} e^{-\beta U}
$$

with $U \equiv U^{p h y s}=U_{h c}+\sum_{i<j} q_{i} q_{j} /\left(D r_{i j}\right)$ the total potential energy and $U_{h c}$ the hard-core contribution, equal to $+\infty$ if two particles overlap, and 0 otherwise (other tempered potentials can be used, but the main results of this section will remain unchanged). If the fluid particles are strongly associated into bound $(+,-)$ pairs, as occurs in the lowtemperature and low-density region in which the vaporliquid transition occurs for the ionic fluids, the "physical" representation described above will be inadequate, and it can be replaced by a "chemical" picture, in which the fluid is composed of associated pairs and free ions. First, a rule that unequivocally identifies bound pairs for each configuration of the ionic fluid (up to a set of null measure in the configurational space) is needed. Once such a rule is defined, we can write the canonical configurational partition function as

$$
Z_{U}\left(N_{i}, N_{i}\right)=\sum_{N_{p}=0}^{N_{i}} Z_{U}^{*}\left(N_{f}, N_{f}, N_{p}\right)
$$

where $N_{p}$ is the number of bound pairs, $N_{f}=N_{i}-N_{p}$ is the number of free ions of each species, and $Z_{U}^{*}\left(N_{f}, N_{f}, N_{p}\right)$ is the canonical configurational partition function corresponding to a system of $N_{p}$ associated pairs and $N_{f}$ free ions of each species,

$$
\begin{aligned}
Z_{U}^{*}\left(N_{f}, N_{f}, N_{p}\right)= & \frac{1}{N_{p} !} \frac{1}{\left(N_{f} !\right)^{2}} \int_{V^{2\left(N_{p}+N_{f}\right)}} d(1) \ldots \\
& \times d\left(N_{p}\right) d \mathbf{r}_{1}^{+} d \mathbf{r}_{1}^{-} \ldots d \mathbf{r}_{N_{f}}^{+} d \mathbf{r}_{N_{f}}^{-} e^{-\beta U^{*}},
\end{aligned}
$$

where $(i) \equiv\left\{\mathbf{r}^{+}, \mathbf{r}^{-}\right\}_{i}$ are the positions of the ions that compose the associated pair $i$, and $\mathbf{r}_{i}^{+(-)}$corresponds to the coordinates of $\mathrm{a}+(-)$ free ion $i$, respectively. The potential energy $U^{*} \equiv U^{\text {chem }}$ does not coincide, in general, with the physical potential energy $U^{\text {phys }}$, since different configurations of the "chemical" mixture can be compatible with a given ionic configuration.

Substituting Eq. (7) into Eq. (5) and rearranging the resulting expression, the neutral grand-canonical partition function can be written as

$$
\Xi=\sum_{N_{f}=0}^{\infty} \sum_{N_{p}=0}^{\infty}\left(\frac{e^{\beta \mu}}{\Lambda_{+}^{3}}\right)^{N_{f}}\left(\frac{e^{\beta \mu}}{\Lambda_{-}^{3}}\right)^{N_{f}}\left(\frac{e^{\beta \mu_{p}}}{\Lambda_{p}^{6}}\right)^{N_{p}} Z_{U}^{*}\left(N_{f}, N_{f}, N_{p}\right)
$$

with $\mu_{p} \equiv 2 \mu$ and $\Lambda_{p}=\sqrt{\Lambda_{+} \Lambda_{-}}$. These last definitions correspond to the chemical equilibrium conditions between the free ions and the associated pairs $[21,36]$. We remark that this derivation is independent of the criterion chosen to define the pairs. However, the choice must be such that matches the microscopic structure of the fluid. As we have seen in the preceding Section, the vapor structure is dominated by neutral aggregates, mostly dimers and tetramers. This fact is consistent with the Bjerrum ideas of pairing [25]. Consequently, a distance-based criterion seems to be the most appropriate: two unlike ions that are closer than $R_{c}\left(R_{c}\right.$ being a suitable cutoff distance) are considered as an associated pair. It is easy to see that such a criterion does not define uniquely the associated pairs when the population of tetramers and higher-order neutral clusters is not negligible, as it can be seen from Fig. 7, since there are different arrangements of associated pairs that are compatible with the same ionic configuration. Hence, a more systematic criterion is needed in order to be able to define associated pairs for $a l$ most every ionic configuration, while keeping the intuitive 


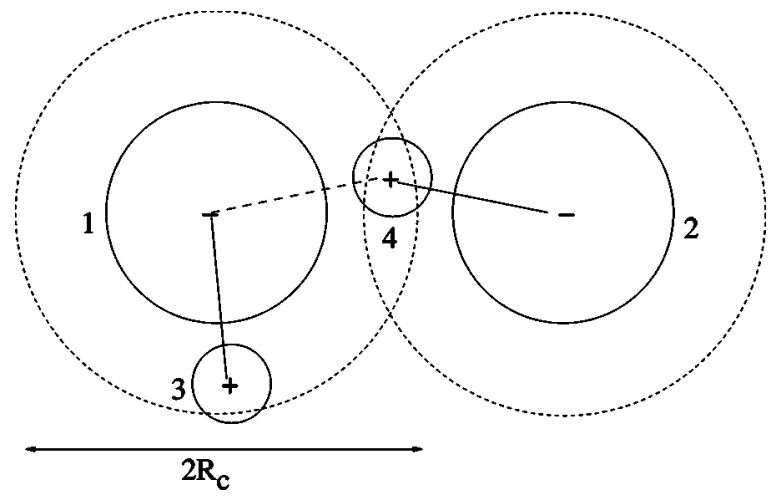

FIG. 7. Different ways of pairing ions using the criterion that two unlike ions are paired if the distance between them is shorter than the cutoff $R_{c}$. The cation 4 can be paired with either the anion 1 or the anion 2. In the first case the ions 2 and 3 remain free, and in the latter case the ions 1 and 3 constitute another associated pair.

definition of an associated pair as encompassing unlike ions that are at the closest distances. We use a suitable modification of the Stillinger-Lovett pair definition on a given ionic configuration [31]. In this prescription, all the distances between two unlike ions are computed, and then the first pair is defined as the two unlike ions at the closest distance. This step is repeated, taking into account only ions that remain unpaired from previous steps for the evaluation of $(+,-)$ distances. We stop when the minimum distance between two unlike ions is greater than $R_{c}$, considering the remaining ions as free ions (no free ions were considered in Ref. [31], and consequently $R_{c} \equiv \infty$ in that case). This protocol provides a unique configuration of associated pairs and free ions for almost each ionic configuration, since the method is ambiguous only in a subset of the ionic configurations in which at least two $(+,-)$ distances are equal, and the measure of such a set is null in the configurational space.

Obviously, different pairing prescriptions can be used. However, this protocol provides an explicit expression for the chemical potential energy $U^{\text {chem }}$,

$$
\begin{aligned}
U^{\text {chem }}= & U^{\text {phys }}+\sum_{i=1}^{N_{p}} v^{p}\left(\left\{\mathbf{r}^{+}, \mathbf{r}^{-}\right\}_{i}\right) \\
& +\sum_{1 \leqslant i<j \leqslant N_{p}} v^{p p}\left(\left\{\mathbf{r}^{+}, \mathbf{r}^{-}\right\}_{i},\left\{\mathbf{r}^{+}, \mathbf{r}^{-}\right\}_{j}\right) \\
& +\sum_{i=1}^{N_{p}} \sum_{j=1}^{N_{f}} v^{p+}\left(\left\{\mathbf{r}^{+}, \mathbf{r}^{-}\right\}_{i}, \mathbf{r}_{j}^{+}\right) \\
& +\sum_{i=1}^{N_{p}} \sum_{j=1}^{N_{f}} v^{p-}\left(\left\{\mathbf{r}^{+}, \mathbf{r}^{-}\right\}_{i}, \mathbf{r}_{j}^{-}\right) \\
& +\sum_{i=1}^{N_{f}} \sum_{j=1}^{N_{f}} v^{+-}\left(\mathbf{r}_{i}^{+}, \mathbf{r}_{j}^{-}\right)
\end{aligned}
$$

where $U^{\text {phys }}$ is the physical potential energy due to the hardcore and eletrostatic interactions, and the other terms correspond to effective interactions of entropic origin needed to reduce the configurational space of the chemical system. The term $v^{p}\left(\left\{\mathbf{r}^{+}, \mathbf{r}^{-}\right\}_{i}\right)$ depends only on the positions of the ions that compose an associated pair and confines them to be at a distance shorter than $R_{c}$,

$$
v^{p}\left(\left\{\mathbf{r}^{+}, \mathbf{r}^{-}\right\}_{i}\right)= \begin{cases}0, & \left|\mathbf{r}_{i}^{+}-\mathbf{r}_{i}^{-}\right| \leqslant R_{c} \\ +\infty & \left|\mathbf{r}_{i}^{+}-\mathbf{r}_{i}^{-}\right|>R_{c}\end{cases}
$$

The pairwise potential energy $v^{p p}\left(\left\{\mathbf{r}^{+}, \mathbf{r}^{-}\right\}_{i},\left\{\mathbf{r}^{+}, \mathbf{r}^{-}\right\}_{j}\right)$ corresponds to a steric hindrance condition between two associated pairs [31], since the distances between two unlike ions corresponding to different pairs cannot be shorter than the minimum distance between the ions that compose each pair,

$$
v^{p p}\left(\left\{\mathbf{r}^{+}, \mathbf{r}^{-}\right\}_{i},\left\{\mathbf{r}^{+}, \mathbf{r}^{-}\right\}_{j}\right)= \begin{cases}+\infty & \left|\mathbf{r}_{i}^{\mp}-\mathbf{r}_{j}^{ \pm}\right| \leqslant d_{i j} \\ 0 & \text { otherwise }\end{cases}
$$

with $d_{i j} \equiv \min \left(\left|\mathbf{r}_{i}^{+}-\mathbf{r}_{i}^{-}\right|,\left|\mathbf{r}_{j}^{+}-\mathbf{r}_{j}^{-}\right|\right)$.

The interaction between the associated pairs and the free ions is modified by the term $v^{p \pm}\left(\left\{\mathbf{r}^{+}, \mathbf{r}^{-}\right\}_{i}, \mathbf{r}_{j}^{ \pm}\right)$, which prevents the free ion from being closer to the unlike ion of the associated pair than its partner,

$$
v^{p \pm}\left(\left\{\mathbf{r}^{+}, \mathbf{r}^{-}\right\}_{i}, \mathbf{r}_{j}^{ \pm}\right)= \begin{cases}+\infty, & \left|\mathbf{r}_{i}^{\mp}-\mathbf{r}_{j}^{ \pm}\right| \leqslant\left|\mathbf{r}_{i}^{+}-\mathbf{r}_{i}^{-}\right| \\ 0, & \text { otherwise }\end{cases}
$$

Finally, two unlike free ions cannot be at a shorter distance than $R_{c}$ due to the term $v^{+-}\left(\mathbf{r}_{i}^{+}, \mathbf{r}_{j}^{-}\right)$,

$$
v^{+-}\left(\mathbf{r}_{i}^{+}, \mathbf{r}_{j}^{-}\right)= \begin{cases}+\infty, & \left|\mathbf{r}_{i}^{+}-\mathbf{r}_{j}^{-}\right| \leqslant R_{c} \\ 0, & \left|\mathbf{r}_{i}^{+}-\mathbf{r}_{j}^{-}\right|>R_{c}\end{cases}
$$

It is not hard to see that a mixture of associated pairs and free ions with a potential energy given by Eq. (10) is completely equivalent to the original ionic system. It is interesting to note that, except the term on $v_{p}$ that ties the ions that compose an associated pair, the other terms are pairwise, short-ranged modifications of the hard-core conditions, so the potential energy of an allowed mixture configuration is the same as in the corresponding ionic configuration. Moreover, every configuration of associated pairs and free ions obtained from an ionic configuration by the pairing procedure described above fulfil the constrains induced by the added potentials. Conversely, the mixture configuration is the same as the one obtained from the corresponding ionic configuration by the Stillinger-Lovett protocol.

This analysis provides an exact chemical representation of the ionic system as a mixture of bound pairs and free ions in chemical equilibrium. The equivalence between representations of the system is not only at the level of thermodynamic properties, but also in the microscopic structure, unlike previous studies based on the matching of the chemical and the physical free energies, e.g., via the virial coefficients [37,38]. In those studies the effective potentials given by Eqs. (11) and (14) could be guessed, but the other terms (that arise from matching third- and higher-order virial coefficients) 


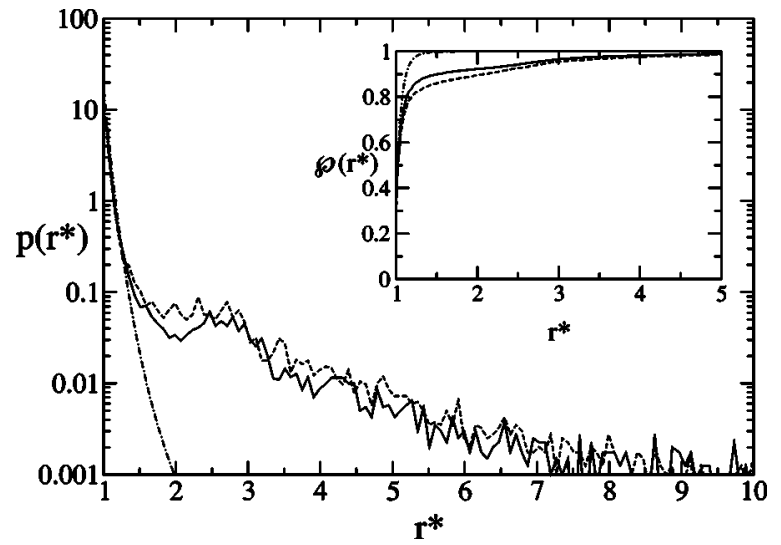

FIG. 8. Probability distribution $p\left(r^{*}\right)$ of having a pair an internal separation distance $r^{*}$ between ions at $T^{*}=0.0443$ and reduced densities $\rho^{*}=0.0064(7)$ (solid line), corresponding to the gas phase, and $\rho^{*}=0.149$ (3) (dashed line), corresponding to the liquid phase. The dot-dashed line corresponds to the ideal dimer fluid approach (see text). The inset shows the cumulant distribution $\wp\left(r^{*}\right) \equiv \int_{1}^{r^{*}} p(t) d t$. The meaning of the symbols is the same as before.

were not clearly identified. Furthermore, they have not been used at all in pairing theories of electrolytes. We must stress the importance of the effective new term given by Eq. (12) in the potential energy, which reduces the configuration space available to the associated pairs. This effect is specially important for high values of $R_{c}$. An extreme case that illustrates the effect of missing the steric hindrance term is the loosely tethered dimer fluid, in which the ions that compose the pair can be at any relative distance greater than $\sigma_{ \pm}$. In this case, the canonical partition function of the dimer fluid is $N_{p} ! Z_{U}\left(N_{p}, N_{p}\right)$, where $N_{p}$ is the number of dimers, $Z_{U}\left(N_{p}, N_{p}\right)$ is given by Eq. (6) and the $N_{p}$ ! factor is the number of different ways of pairing the unlike ions in each ionic configuration and lead to different dimer configurations. As a consequence, in the thermodynamic limit the Helmholtz free energy per particle $f_{d}$ of the loosely tethered dimer fluid diverges as

$$
\frac{f_{d}}{k_{B} T} \sim-\ln N_{p}+\left(\frac{2 f_{i}}{k_{B} T}+1\right),
$$

where $f_{i}$ is the ionic Helmholtz free energy per ion, which is well behaved in the case of neutral systems [35].

The association degree of the ionic fluid in the coexistence region has been studied by computer simulation in the framework of the previous analysis. In the usual grandcanonical simulations, we have identified the associated pairs by the Stillinger-Lovett rule with $R_{c}=\sqrt{3} L / 2$, i.e., all the ions are associated, and the minimum image convention is used in order to calculate the distances between unlike ions. The probability distributions $p(r)$ of a pair having an internal separation distance $r$ between ions is plotted in Fig. 8 for the gas and liquid phases at $T^{*}=0.0443$. Surprisingly, both distributions are practically identical, despite the fact that the corresponding densities are quite different. The distributions

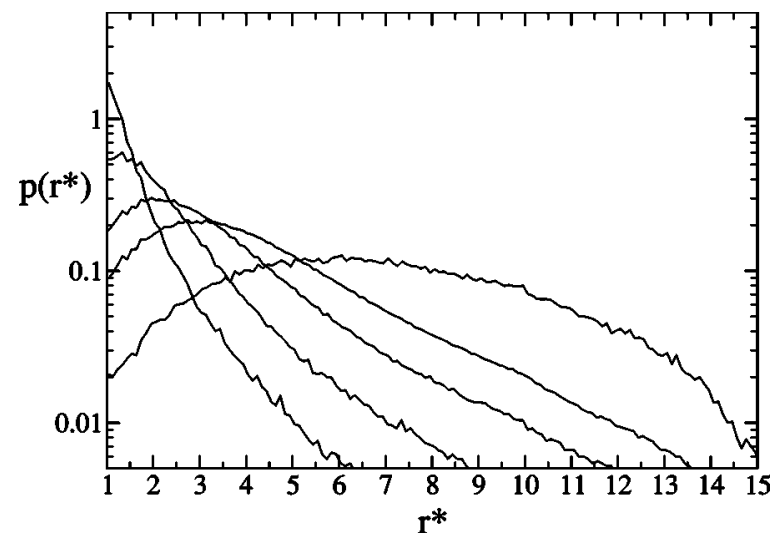

FIG. 9. Probability distribution $p\left(r^{*}\right)$ at $T^{*}=1$. At $r^{*}=1$, from bottom to top the lines correspond to reduced densities $\rho^{*}$ $=0.00113(1), 0.00618(1), 0.01453(2), 0.0456(3)$, and 0.1566(6).

show a very pronounced peak for $r=\sigma_{ \pm}$, and a local maximum around $r=2.5 \sigma_{ \pm}$, approximately where $g_{+-}$presents the second local maximum. For large values of $r$, the distributions $p(r)$ decay to zero. These results confirm the strong association in the ionic fluid at low temperatures, and that the structure is weakly affected by variations in density, at least in the range in which the gas-liquid coexistence occurs. For values of $r$ close to the contact value, the distribution is well described by the noninteracting pair fluid probability distribution, given by the following expression:

$$
\begin{aligned}
p^{\text {ideal }}(r) & \approx \frac{r^{2} \exp \left(\frac{q^{2}}{D k_{B} T r}\right)}{\int_{\sigma_{ \pm}}^{R_{c}} y^{2} \exp \left(\frac{q^{2}}{D k_{B} T y}\right) d y} \\
& \sim \frac{q^{2} r^{2}}{D k_{B} T \sigma_{ \pm}^{4}} \exp \left(\frac{q^{2}}{D k_{B} T \sigma_{ \pm}}\left[\frac{\sigma_{ \pm}}{r}-1\right]\right)
\end{aligned}
$$

valid for $T^{*} \ll 1$ and an internal cutoff $R_{c} \gg \sigma_{ \pm}$. However, for $r \gtrsim 1.5 \sigma_{ \pm}, p(r)$ deviates from the ideal expression as a consequence of the interaction between associated pairs. The local maximum showed by the distribution function indicates that the most bound pairs are solvated by less bound pairs, to form stable neutral tetramers (and higher order clusters, but their inclusion does not seem to affect the conclusions of this section). This fact is in agreement with the features observed in the pair correlation functions in the preceding section. We must stress that the structure that $p(r)$ presents is only due to the Coulombic interactions.

It is instructive to compare our results in the coexistence region to the higher temperature ones. We have computed $p(r)$ at $T^{*}=1$ and the same range of densities (see Fig. 9). The qualitative behavior of $p(r)$ at high temperatures is similar to that predicted by Stillinger and Lovett [39]. First, it shows a maximum localized in $r \sim \rho^{-1 / 3}$. On the other hand, $p(r)$ takes significant values in a wider range than the corresponding functions at lower temperatures, decaying for 
large values of $r$ as $r^{-n}$, with $n \approx 3$. The latter prediction differs from the value $n=4$ given in Ref. [39] and requires further study to elucidate it.

The probability distribution $p^{R_{c}}(r)$ of a pair having an internal separation distance between ions when the cutoff distance to define a pair is equal to $R_{c}$ can be obtained from $p(r)$ as

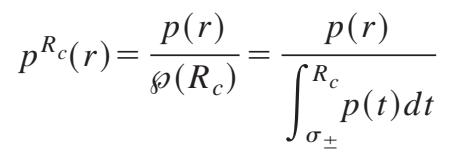

where $\wp(r) \equiv \int_{\sigma_{ \pm}}^{r} p(t) d t$ is the cumulant distribution corresponding to $p(r)$. The density of associated pairs is equal to $\rho \wp\left(R_{c}\right) / 2$, and the total density of free ions is $\rho[1$ $\left.-\wp\left(R_{c}\right)\right]$, where $\rho$ is the total density of ions in the physical picture. It can be seen from Fig. 8 that for $R_{c} \gtrsim 3 \sigma_{ \pm}$, more than $95 \%$ of ions are associated into pairs in both vapor and liquid phases. So, if the associated pair fluid phase separate in the same $(T, \rho)$ region as the ionic fluid, it is expected that the free ions do play a mere perturbative role in the phase coexistence. Actually, the free ionic subsystem is a nonadditive binary charged hard-sphere mixture, where $R_{c}$ plays the role of the unlike-ion collision diameter. However, the interactions between like ions are purely repulsive, and we do not expect differences between the behavior of this mixture and the RPM as soon as $\sigma_{+}$and $\sigma_{-}$are less than $R_{c}$. Furthermore, this system is in a polar environment given by the associated pair subsystem, and consequently the effective dielectric constant $D_{\text {eff }}$ of the background will be increased. Then the free ion subsystem is expected to have the same behavior as the RPM at the effective reduced temperature $\widetilde{T}$

$$
\widetilde{T}=\frac{D_{e f f} k_{B} T R_{c}}{q^{2}}=\frac{D_{e f f}}{D} \frac{R_{c}}{\sigma_{ \pm}} T^{*},
$$

where $T^{*}$ is the reduced temperature. For $R_{c} \gtrsim 3 \sigma_{ \pm}$, the critical temperature reduces at least to one-third of the RPM reduced critical temperature. Consequently, any possible free ion-driven vapor-liquid transition should occur at much lower temperatures, and thus the free ion subsystem should play no role in the vapor-liquid transition. In order to check this hypothesis, the phase diagram of the associated pair fluid has to be obtained. This issue will be addressed in the following section.

\section{PHASE BEHAVIOR OF THE ASSOCIATED PAIRS FLUID}

The results obtained in the preceding section suggest that the chemical picture introduced above is a very convenient description of the ionic fluid structure. Furthermore, we can analyze the role played by the associated pairs in the phase equilibrium by eliminating the free ions. We have performed grand-canonical Monte Carlo simulations of the associated dimer system for $\lambda=3$ and different values of $R_{c}$. No free ions are allowed $\left(N_{f} \equiv 0\right)$ and the potential energy of one configuration is given by Eq. (10). The grand-canonical free energies corresponding to these systems will consequently provide an upper bound to the ionic fluid grand-canonical free energy at the same temperature and pair chemical potential. As for the ionic system, a fine-discretization approach with a refinement parameter $\zeta=10$, and Ewald summation technique with conducting boundary conditions is used to take into account the long range character of the Coulombic interations. The basic steps are either insertion or deletion of associated pairs (chosen randomly with the same probability), biased with a Boltzmann distribution that depends on the separation between the ions that compose the dimer. An associated pair is inserted in the following way: the negative ion is placed at a random place in the box, and its counterion is placed at a relative position $\mathbf{r}_{ \pm}\left(\sigma_{ \pm} \leqslant\left|\mathbf{r}_{ \pm}\right|<R_{c}\right)$ following a probability distribution proportional to $\exp \left[\beta_{0} q^{2} \phi_{0}\left(\mathbf{r}_{ \pm}\right)\right]$, where $-\phi_{0}(\mathbf{r})$ is the Ewald potential energy between two unlike ions of unit charge. On the other hand, a pair is deleted with a probability proportional to $\exp \left[\beta_{0} q^{2} \phi_{0}\left(\mathbf{r}_{ \pm}\right)\right], \mathbf{r}_{ \pm}$ being the relative position of the positive ion of the pair with respect to the negative one. The value of $\beta_{0}$ has been adjusted to improve the sampling during the simulation. In this work we have set $\beta_{0}=8$.

The acceptance probabilities of a pair insertion $W_{i j}^{i}$ and a pair deletion, $W_{i j}^{d}$ are the following:

$$
\begin{aligned}
W_{i j}^{i}= & \min \left(1,\left(L^{*}\right)^{3} \exp (\beta \mu-\beta \Delta U)\right. \\
& \times\left[\sum_{\sigma_{ \pm} \leqslant \mathbf{r}_{ \pm} \mid<R_{c}} \frac{\exp \left[\beta_{0} q^{2} \phi_{0}\left(\mathbf{r}_{ \pm}\right)\right]}{\zeta^{3}}\right] \\
& \left.\times\left[\sum_{k=1}^{N_{p}+1} \exp \left[\beta_{0} q^{2} \phi_{0}\left(\mathbf{r}_{ \pm}^{k}\right)\right]\right]^{-1}\right), \\
W_{i j}^{d}= & \min \left(1, \frac{\exp (-\beta \mu-\beta \Delta U)}{\left(L^{*}\right)^{3}}\right. \\
& \times\left[\sum_{\sigma_{ \pm} \leqslant \mathbf{r}_{ \pm} \mid<R_{c}} \frac{\exp \left[\beta_{0} q^{2} \phi_{0}\left(\mathbf{r}_{ \pm}\right)\right]}{\zeta^{3}}\right]^{-1} \\
& \left.\times\left[\sum_{k=1}^{N_{p}} \exp \left[\beta_{0} q^{2} \phi_{0}\left(\mathbf{r}_{ \pm}^{k}\right)\right]\right]\right)
\end{aligned}
$$

where $i$ and $j$ are the initial and final configurations, respectively, $N_{p}$ is the number of pairs at the configuration $i, \beta$ $=1 /\left(k_{B} T\right), \mu$ is the configurational chemical potential, and $\Delta U$ is the chemical potential energy variation in the movement, including the steric hindrance terms given by Eq. (12). As it can be seen, the acceptance probabilities reduce to the usual ones as $\beta_{0} \rightarrow 0$.

Effective critical points for different values of $L^{*}$ were estimated by using mixed-field finite-size scaling methods [34] and assuming Ising-like criticality. Although recent results [40] indicate that the pressure should also enter the field mixing, our approach should be satisfactory to discern the dependence of the critical parameters on $R_{c}$. Moreover, a 


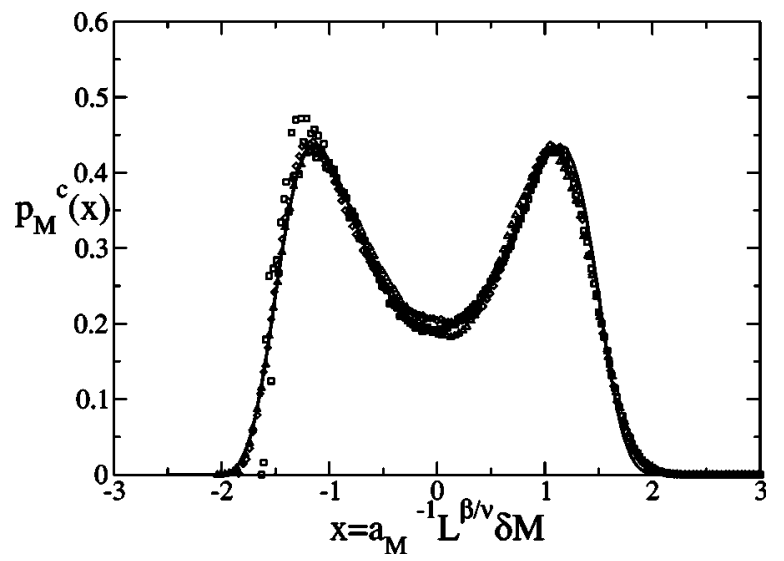

FIG. 10. Rescaled marginal probability distributions $p_{\mathcal{M}}^{c}(x)$ for the associated pairs fluid characterized by $\lambda=3$ and $R_{c}^{*}=3$. The solid line is the universal function corresponding to the threedimensional Ising universality class, and the symbols correspond to the best-matching simulation results: the squares correspond to $L^{*}$ $=12$, the diamonds to $L^{*}=15$, and the triangles to $L^{*}=18$.

systematic study for the RPM case has shown that the assumed universality class is very likely to be the right one [16]. We use histogram reweighting techniques [33] to combine the histograms from different runs (typically three) and estimate the critical parameters and their standard errors. Very long runs are needed in order to overcome the critical slowing down and the low acceptance ratios due to the low temperatures involved. Defining a step as a try of a pair insertion (or deletion), we have performed simulations of about $\left(2 \times 10^{7}\right)-\left(4 \times 10^{7}\right)$ equilibration steps and $(2$ $\left.\times 10^{8}\right)-\left(4 \times 10^{8}\right)$ sampling steps for $L^{*}=12 ;\left(5 \times 10^{7}\right)-(2$ $\left.\times 10^{8}\right)$ equilibration steps and $\left(6 \times 10^{8}\right)-\left(1.2 \times 10^{9}\right)$ sampling steps for $L^{*}=15$; and $2 \times 10^{8}$ equilibration steps and $\left(1.2 \times 10^{9}\right)-\left(1.8 \times 10^{9}\right)$ sampling steps for $L^{*}=18$, in order to get good statistics for the histograms. The (effective) critical parameters are obtained by minimizing the deviation of the appropriately scaled mixed-field $\mathcal{M} \propto \rho-s u$ marginal probability distribution ( $u$ is the potential energy density and $s$ is the mixing parameter) with respect to the corresponding critical three-dimensional Ising universal function [34,41]. As also occurs in the ionic and tethered dimer systems, the matching improves as $L^{*}$ increases (see Fig. 10). Our estimations of the critical parameters for different values of $L^{*}$ and $R_{c}^{*} \equiv R_{c} / \sigma_{ \pm}$are listed in Table I and plotted in Figs. 11 and 12 . The smallest value of $R_{c}^{*}$ is the same as the maximum allowed separation in the tethered dimer system, and as expected the results obtained for the associated pairs fluid are practically indistinguishable from the tethered dimer system. As $R_{c}^{*}$ increases, we observe a sharp increase in the critical temperature but the critical density remains almost unaffected. However, for $R_{c}^{*} \approx 3-4$ the critical temperature reaches a maximum and then decreases, converging towards the free ion critical temperature. On the other hand, the critical density also decreases towards the free ion critical density, although the statistical uncertainties are bigger for this parameter and the exact path of convergence is less defined.
TABLE I. Dependence on $R_{c}^{*}$ of the estimated critical parameters. (The $1 \sigma$ statistical uncertainties refer to the last decimal places.)

\begin{tabular}{|c|c|c|c|c|}
\hline$R_{c}^{*}$ & $L^{*}$ & $T_{c}^{*} \times 10^{2}$ & $-\mu_{c}^{*}$ & $\rho_{c}^{*} \times 10^{2}$ \\
\hline \multirow[t]{3}{*}{1.02} & 12 & $4.24(2)$ & $1.2512(4)$ & $7.66(12)$ \\
\hline & 15 & $4.25(1)$ & $1.2504(2)$ & $7.56(9)$ \\
\hline & 18 & $4.26(1)$ & $1.25077(8)$ & $7.34(10)$ \\
\hline \multirow[t]{3}{*}{1.5} & 12 & $4.44(1)$ & $1.30489(10)$ & $7.41(12)$ \\
\hline & 15 & $4.46(1)$ & $1.30565(5)$ & $7.52(10)$ \\
\hline & 18 & $4.47(1)$ & $1.30580(8)$ & $7.33(17)$ \\
\hline \multirow[t]{3}{*}{2.0} & 12 & $4.53(2)$ & $1.3112(3)$ & $7.45(14)$ \\
\hline & 15 & $4.53(1)$ & $1.3109(2)$ & $7.53(18)$ \\
\hline & 18 & $4.53(2)$ & $1.3107(3)$ & $7.54(17)$ \\
\hline \multirow[t]{3}{*}{3.0} & 12 & $4.65(2)$ & $1.3187(4)$ & $7.23(10)$ \\
\hline & 15 & $4.60(1)$ & $1.31792(13)$ & $6.93(12)$ \\
\hline & 18 & $4.62(3)$ & $1.3182(4)$ & $6.69(10)$ \\
\hline \multirow[t]{3}{*}{4.0} & 12 & $4.56(4)$ & $1.3202(5)$ & $7.05(22)$ \\
\hline & 15 & $4.59(2)$ & $1.3207(5)$ & $6.45(14)$ \\
\hline & 18 & $4.62(3)$ & $1.3213(5)$ & $6.4(2)$ \\
\hline \multirow[t]{3}{*}{5.0} & 12 & $4.58(1)$ & $1.32210(14)$ & $6.17(21)$ \\
\hline & 15 & $4.57(1)$ & $1.32230(7)$ & $6.46(24)$ \\
\hline & 18 & $4.56(4)$ & $1.3220(7)$ & $6.17(10)$ \\
\hline \multirow[t]{3}{*}{6.0} & 12 & $4.55(1)$ & $1.3222(3)$ & $6.41(2)$ \\
\hline & 15 & $4.51(2)$ & $1.3214(3)$ & $6.33(10)$ \\
\hline & 18 & $4.59(3)$ & $1.3222(6)$ & $6.1(2)$ \\
\hline \multirow[t]{2}{*}{7.0} & 15 & $4.51(2)$ & $1.3216(5)$ & $6.33(10)$ \\
\hline & 18 & $4.50(2)$ & $1.3211(3)$ & $6.04(17)$ \\
\hline 8.0 & 18 & $4.53(4)$ & $1.3224(6)$ & $6.1(4)$ \\
\hline 9.0 & 18 & $4.49(1)$ & $1.3215(3)$ & $5.6(3)$ \\
\hline \multirow[t]{3}{*}{ Free ion case ${ }^{a}$} & 12 & $4.49(1)$ & $1.3200(1)$ & $6.37(7)$ \\
\hline & 15 & $4.48(1)$ & $1.3199(1)$ & $6.04(11)$ \\
\hline & 18 & $4.49(2)$ & $1.3199(4)$ & $6.05(11)$ \\
\hline
\end{tabular}

${ }^{\text {a It }}$ is equivalent to consider $R_{c}^{*} \equiv \sqrt{3} L^{*} / 2$.

We must also point out that the statistical uncertainties are big enough not to make possible an estimation of correctionto-scaling effects.

For a heuristic explanation for such a behavior, we have to consider the energetically favored configurations. It is important to consider the interaction between two different associated pairs, which has its minimum energy configuration in a square ring conformation for low values of $\lambda$, with a close-energy secondary minimum conformation for the linear $(+,-)$ chain [17]. If $R_{c}$ is too small, by deforming the square it is possible to find energetically relevant configurations (i.e., the potential energy difference per ion of such configurations with respect to the minimum one is of the same order as the thermal energy) in which one of the associated pairs has an internal ionic separation less than $R_{c}$, but the other one does not fulfil such a condition. Consequently, a higher value of $R_{c}$ also increases the number of energetically relevant configurations between two associated pairs. Then the effective interaction between associated pairs is enhanced, and thus an increase of the critical temperature should be expected until all the relevant configurations are allowed (that occurs for $R_{c} \sim 3 \sigma_{ \pm}$). On the other hand, the 


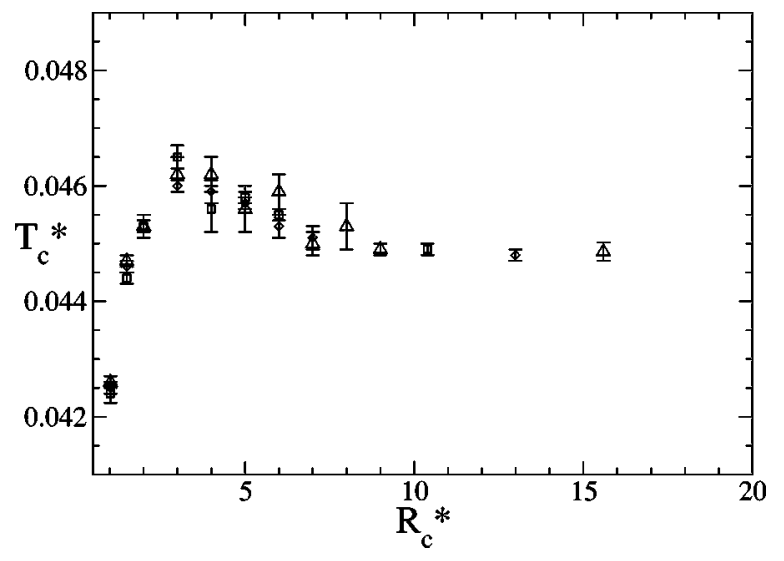

FIG. 11. Dependence of the critical temperature $T_{c}^{*}$ on the reduced cutoff distance $R_{c}^{*}$, for $L^{*}=12$ (squares), $L^{*}=15$ (diamonds), and $L^{*}=18$ (triangles).

average size of the associated pairs and their aggregates is increased, leading to a decrease of the critical density (in order to keep the reduced density in terms of the effective particle size constant). These arguments are no longer valid for $R_{c}^{*} \geq 3$. Our results show that the inclusion of associated pairs with larger internal ionic separations is not crucial to the gas-liquid phase transition. Also they bar the phase transition, as can be inferred from the critical parameters decrease.

As for the ionic and tethered dimer models, histogram reweighting techniques $[33,41]$ allow us to obtain the coexistence curve up to $T \leqslant 0.98 T_{c}$. As the finite-size effects are not important far from the critical point, we have considered $L^{*}=15$. Taking advantage of the wide range of densities covered by near-critical histograms, we combine them with liquid subcritical state histograms in order to extend the density range. The extra simulations involve shorter runs (typically $2 \times 10^{8}$ steps after equilibration). The gas-liquid coexistence curves for different values of $R_{c}^{*}$ are plotted in Fig.

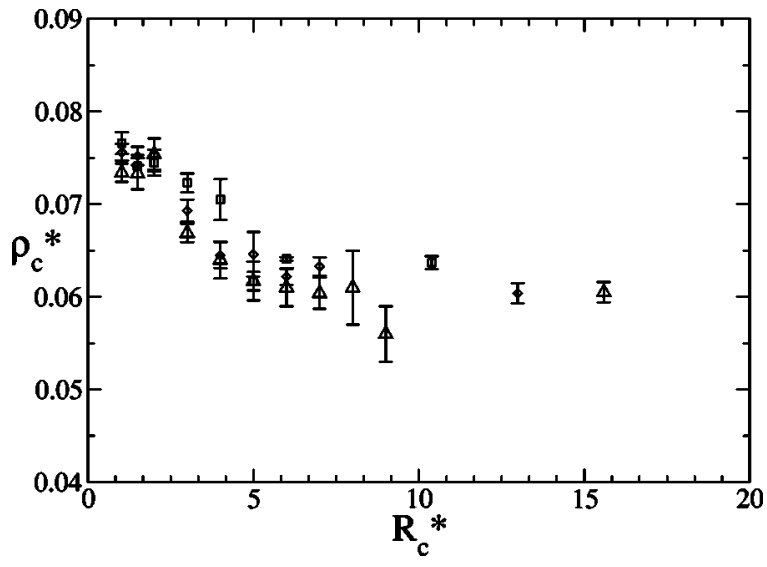

FIG. 12. Dependence of the critical density $\rho_{c}^{*}$ on the reduced cutoff distance $R_{c}^{*}$, for $L^{*}=12$ (squares), $L^{*}=15$ (diamonds), and $L^{*}=18$ (triangles).

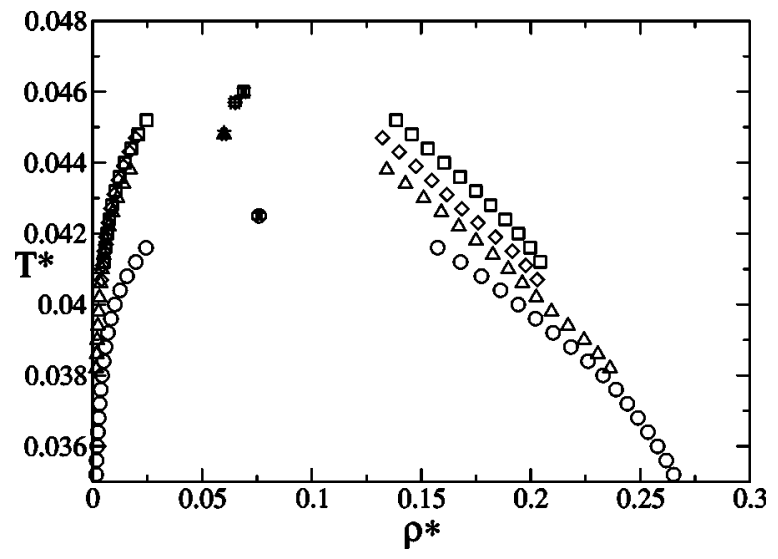

FIG. 13. Gas-liquid coexistence curves for $R_{c}^{*}=1.02$ (circles), $R_{c}^{*}=3$ (squares), $R_{c}^{*}=5$ (diamonds), and the free ion system (triangles).

13, showing the same tendency as the observed one in the critical parameters. It is interesting to note that the vapor branches coincide (except close to the critical point) for $R_{c}$ $>3 \sigma_{+}$. We conclude from this observation that is in the liquid branch where the ionic fluid differs mostly from the associated pair system.

\section{DISCUSSION AND CONCLUSIONS}

In summary, an exact "chemical" representation of the ionic system as a mixture of $(+,-)$ associated ion pairs and free ions has been introduced. This representation, closely related to the Stillinger-Lovett pairing procedure for the RPM, has the advantage of not only providing an exact matching of the physical and chemical representation thermodynamics, from a microscopic point of view; it also avoids an entropy catastrophe that occurs for the tethered dimer model studied at large values of the tether length. The addition to the physical Hamiltonian of some new pairwise hard-core interactions between the chemical components provides a suitable Hamiltonian for the chemical representation. In the low temperature and low density regime, in which the ionic vapor-liquid transition occurs, such a representation provides a faithful characterization of the microscopic structure of the ionic fluid, and it can be the basis of improved theoretical approaches.

The analysis of the phase behavior of the system only composed of associated pairs, indicates strongly that the ionic fluid vapor-liquid transition is driven by them. For $R_{c}$ $\lesssim 3 \sigma_{ \pm}$, the critical temperature increases with $R_{c}$, as more energetically favored configurations are allowed. For $R_{c}$ $\geq 3 \sigma_{ \pm}$, the critical parameters converge smoothly from above towards the ionic fluid critical parameters as $R_{c}$ increases. This fact indicates not only that the free ions do not drive the phase transition, but also they have the opposite effect in the transition. The value of $R_{c} \approx 3 \sigma_{ \pm}$corresponding to the maximum on the critical temperature, can be regarded as the optimal size of the associated pair.

Some remarks on the limitations of the present work are 
appropriate at this point. We have focused only on the effect of the associated pairs in the thermodynamical properties. However, the conducting character of the ionic fluid is completely driven by the free ions. On the other hand, the remarkable success of pairing theories (even when the "chemical" representation they implicitly use is not completely correct) remains unexplained. It is possible that the associated pair solvation, in addition to the ion-ion correlations, can mimic the pair-pair interactions. Further studies are needed to completely solve the origin of the ionic fluid vapor-liquid phase transition.

\section{ACKNOWLEDGMENTS}

J.M.R.-E. and L.F.R. gratefully acknowledge financial support for this research by Grant No. PB97-0712 from DGICyT (Spain) and No. FQM-205 from PAI (Junta de Andalucía). J.M.R.-E also wishes to thank Ministerio de Educación, Cultura y Deporte (Spain) for partial financial support. A.Z.P. acknowledges financial support from the Department of Energy (Grant No. DE-FG02-01ER1512) and ACS-PRF (Grant No. 38165-AC9).
[1] M.E. Fisher, J. Stat. Phys. 75, 1 (1994); J. Phys.: Condens. Matter 8, 9103 (1996).

[2] R.R. Singh and K.S. Pitzer, J. Chem. Phys. 92, 6775 (1990).

[3] H. Weingärtner, S. Wiegand, and W. Schröer, J. Chem. Phys. 96, 848 (1992).

[4] K.C. Zhang, M.E. Briggs, R.W. Gammon, and J.M.H. Levelt Sengers, J. Chem. Phys. 97, 8692 (1992).

[5] T. Narayanan and K.S. Pitzer, Phys. Rev. Lett. 73, 3002 (1994); J. Phys. Chem. 98, 9170 (1994).

[6] J. Jacob, A. Kumar, M.A. Anisimov, A.A. Povodyrev, and J.V. Sengers, Phys. Rev. E 58, 2188 (1998).

[7] M.A. Anisimov, J. Jacob, A. Kumar, V.A. Agayan, and J.V. Sengers, Phys. Rev. Lett. 85, 2336 (2000).

[8] K. Gutkowski, M.A. Anisimov, and J.V. Sengers, J. Chem. Phys. 114, 3133 (2001).

[9] G. Stell, K.C. Wu, and B. Larsen, Phys. Rev. Lett. 37, 1369 (1976)

[10] P.N. Vorontsov-Vel'yaminov and V.P. Chasovskikh, High Temp. 13, 1071 (1975).

[11] G. Orkoulas and A.Z. Panagiotopoulos, J. Chem. Phys. 101, 1452 (1994).

[12] J.M. Caillol, D. Levesque, and J.J. Weis, J. Chem. Phys. 107, 1565 (1997).

[13] Q. Yan and J.J. de Pablo, J. Chem. Phys. 111, 9509 (1999).

[14] A.Z. Panagiotopoulos and S.K. Kumar, Phys. Rev. Lett. 83, 2981 (1999).

[15] A.Z. Panagiotopoulos, J. Chem. Phys. 116, 3007 (2002).

[16] E. Luijten, M.E. Fisher, and A.Z. Panagiotopoulos, Phys. Rev. Lett. 88, 185701 (2002).

[17] J.M. Romero-Enrique, G. Orkoulas, A.Z. Panagiotopoulos, and M.E. Fisher, Phys. Rev. Lett. 85, 4558 (2000).

[18] Q. Yan and J.J. de Pablo, J. Chem. Phys. 114, 1727 (2001); J. Chem. Phys. 86, 2054 (2001).

[19] A.Z. Panagiotopoulos and M.E. Fisher, Phys. Rev. Lett. 88, 045701 (2002).

[20] Q. Yan and J.J. de Pablo, Phys. Rev. Lett. 88, 095504 (2002); J. Chem. Phys. 116, 2967 (2002).
[21] Y. Levin and M.E. Fisher, Physica A 225, 164 (1996).

[22] E. Gonzalez-Tovar, Mol. Phys. 97, 1203 (1999).

[23] E. Gonzalez-Tovar and C.W. Outhwaite, Mol. Phys. 83, 1273 (1994); A.K. Sabir, L.B. Bhuiyan, and C.W. Outhwaite, ibid. 93, 405 (1998).

[24] F.O. Raineri, J.P. Routh, and G. Stell, J. Phys. IV 10, 99 (2000).

[25] N. Bjerrum, Mat. Fys. Medd. K. Dan. Vidensk. Selsk. 7, 1 (1926).

[26] M.J. Gillan, Mol. Phys. 49, 421 (1983).

[27] F. Bresme, E. Lomba, J.J. Weis, and J.L.F. Abascal, Phys. Rev. E 51, 289 (1995).

[28] J.C. Shelley and G.N. Patey, J. Chem. Phys. 103, 8299 (1995).

[29] Y.V. Kalyuzhni, Mol. Phys. 94, 735 (1998).

[30] J. Jiang, L. Blum, and O. Bernard, Mol. Phys. 99, 1765 (2001); J. Jiang, L. Blum, O. Bernard, J.M. Prausnitz, and S.I. Sandler, J. Chem. Phys. 116, 7977 (2002).

[31] F.H. Stillinger and R. Lovett, J. Chem. Phys. 48, 3858 (1968).

[32] G. Hummer, Chem. Phys. Lett. 235, 297 (1995).

[33] A.M. Ferrenberg and R.H. Swendsen, Phys. Rev. Lett. 61, 2635 (1988); 63, 1195 (1989).

[34] N.B. Wilding and A.D. Bruce, J. Phys.: Condens. Matter 4, 3087 (1992).

[35] E.H. Lieb and J.L. Lebowitz, Adv. Math. 9, 316 (1972).

[36] D. Chandler and L.R. Pratt, J. Chem. Phys. 65, 2925 (1976).

[37] T. L. Hill, Statistical Mechanics (McGraw-Hill, New York, 1956), Sec. 27.

[38] M.E. Fisher and D.M. Zuckerman, Chem. Phys. Lett. 293, 461 (1998); M.E. Fisher and D.M. Zuckerman, J. Chem. Phys. 109, 7961 (1998).

[39] F.H. Stillinger and R. Lovett, J. Chem. Phys. 48, 3869 (1968).

[40] M.E. Fisher and G. Orkoulas, Phys. Rev. Lett. 85, 696 (2000); G. Orkoulas, M.E. Fisher, and C. Üstün, J. Chem. Phys. 113, 7530 (2000).

[41] A.Z. Panagiotopoulos, J. Phys.: Condens. Matter 12, R25 (2000). 